\title{
UCRL-TR-209623
}

LAW RENCE LIVERMORE N A T IO N A L LABORATORY
Proton Radiography of Laser-Plasma Interactions with Picosecond Time Resolution

A. J. Mackinnon, P. K. Patel, R. P. J. Town, S. P. Hatchett, D. Hicks, T. H. Phillips, S. C. Wilks, D. Price, M. H. Key, B. Lasinski, B. Langdon, M. Borghesi, L. Romagnani, S. Kar, O.Willi, J. C. Gauthier, M. Koenig, S. Lepape, T. Boehlly

February 11, 2005 
This document was prepared as an account of work sponsored by an agency of the United States Government. Neither the United States Government nor the University of California nor any of their employees, makes any warranty, express or implied, or assumes any legal liability or responsibility for the accuracy, completeness, or usefulness of any information, apparatus, product, or process disclosed, or represents that its use would not infringe privately owned rights. Reference herein to any specific commercial product, process, or service by trade name, trademark, manufacturer, or otherwise, does not necessarily constitute or imply its endorsement, recommendation, or favoring by the United States Government or the University of California. The views and opinions of authors expressed herein do not necessarily state or reflect those of the United States Government or the University of California, and shall not be used for advertising or product endorsement purposes.

This work was performed under the auspices of the U.S. Department of Energy by University of California, Lawrence Livermore National Laboratory under Contract W-7405-Eng-48. 
FY04 LDRD Final Report

\section{Proton Radiography of Laser-Plasma interactions with Picosecond Time resolution}

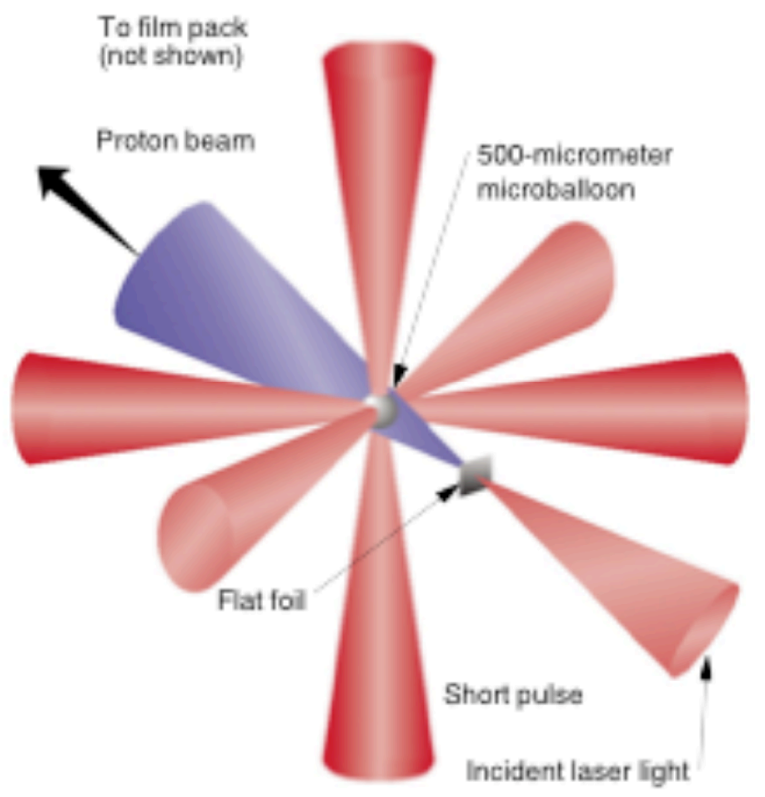

Proton image of asymmetrically imploded core

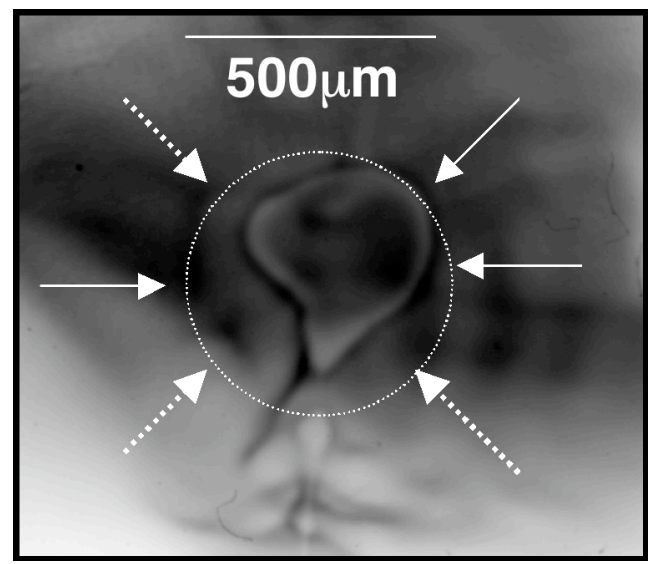

\section{LDRD Project Tracking Code: 02-ERD-012}

Principal Investigator: A.J.Mackinnon

Co investigators LLNL : P.K.Patel R.P.J.Town

Collaborators LLNL: S.P.Hatchett, D.Hicks,T.H.Phillips, S.C.Wilks, D. Price,M.H.Key, B.Lasinski, B. Langdon

Queens University, Belfast: M. Borghesi, L Romagnani, S. Kar

Heinerich Hiene University, Dusseldorf: O. Willi

Ecole Polytechnique: J.C Gauthier, M.Koenig, S.Lepape Laboratory for Laser Energetics: T. Boehlly 
Radiography of laser-produced plasmas with $\mathrm{MeV}$ protons has the potential to provide new information on plasma conditions in extreme states of matter. Protons with energies up to many hundreds $\mathrm{MeV}$, produced by large scale accelerators have been recently been used to obtain mass density radiographs of the behavior of large samples which have been shocked on microsecond timescales with approximately $\mathrm{mm}$ spatial resolution [1]. The recent discovery of laminar proton beams accelerated to multi-MeV energies by picosecond duration laser beams [2] has provided the opportunity to probe dense plasmas with hitherto unparalleled temporal and spatial resolution $[3,4]$.

The object of this research project was to develop the proton radiography technique so that it could be used as a tool to diagnose density conditions and electromagnetic fields in conditions relevant for inertial confinement fusion (ICF) can only be reached on large scale high energy systems such as NIF. The first step to achieving this aim was to investigate the physical mechanisms behind the proton acceleration mechanism. We then studied the spatial resolution of the technique and used experimental data to benchmark a Monte- Carlo code that modeled radiography of dense objects. These techniques were then applied to a scaled down NIF implosion. This dynamic and complex experiment, carried out at the Rutherford Appleton Laboratory allowed us to demonstrate the utility of the proton radiographic technique in an environment that provided similar challenges to diagnosing a full scale NIF implosion.

In a parallel effort we also investigated techniques to measure transient electromagnetic fields in laser-produced plasmas. In this very successful experiment carried out on the LULI laser at Ecole Polytechnique we developed a new proton diagnostic technique, deflectometry, to quantitatively characterize the proton deflections in a plasma generated by a separate laser pulse. These results then allowed us to develop a predictive modeling using two sophisticated simulation codes, Lasnex and LSP. These models are now being used by the ICF program to study magnetic fields in plasmas relevant for (ICF), including NIF scale hohlraums. This project has thus completely achieved all the original objectives. Moreover, the collaborations partially funded by this work have already resulted in the publication of 13 papers including five Physical Review Letters, three in Review of Scientific instruments, two Applied physics letters, one physics of plasmas, one laser and particle beams and one nuclear fusion paper. Two more papers are currently being prepared for submission to Physical Review Letters and Science respectively.

The first part of the proposal required us to investigate if we could control and manipulate the proton beam. To facilitate this we carried out a number of experiments on the JanUSP facility at LLNL to investigate the proton production mechanisms. The JanUSP laser system produced $10 \mathrm{~J}$ in a $100 \mathrm{fs}$ pulse that could be focused to a peak intensity of $1-5 \times 10^{20} \mathrm{Wcm}^{-2}$. By comparing the first experiment results to detailed $2 \mathrm{D}$ PIC simulations we discovered that high energy electrons, produced when the intense laser pulse is absorbed at the solid target surface, reflux repeatedly throughout the target enhancing the acceleration of protons at the rear (and front) target surface. Figure 1 shows how the proton energy increases due to a refluxing population of electrons inside the target. 
This mechanism is only effective while the laser pulse is still on and therefore no enhancement occurs when the target is thicker than the round trip time of an electron inside the target ( 15 microns for a 100fs laser pulse). This work was published in

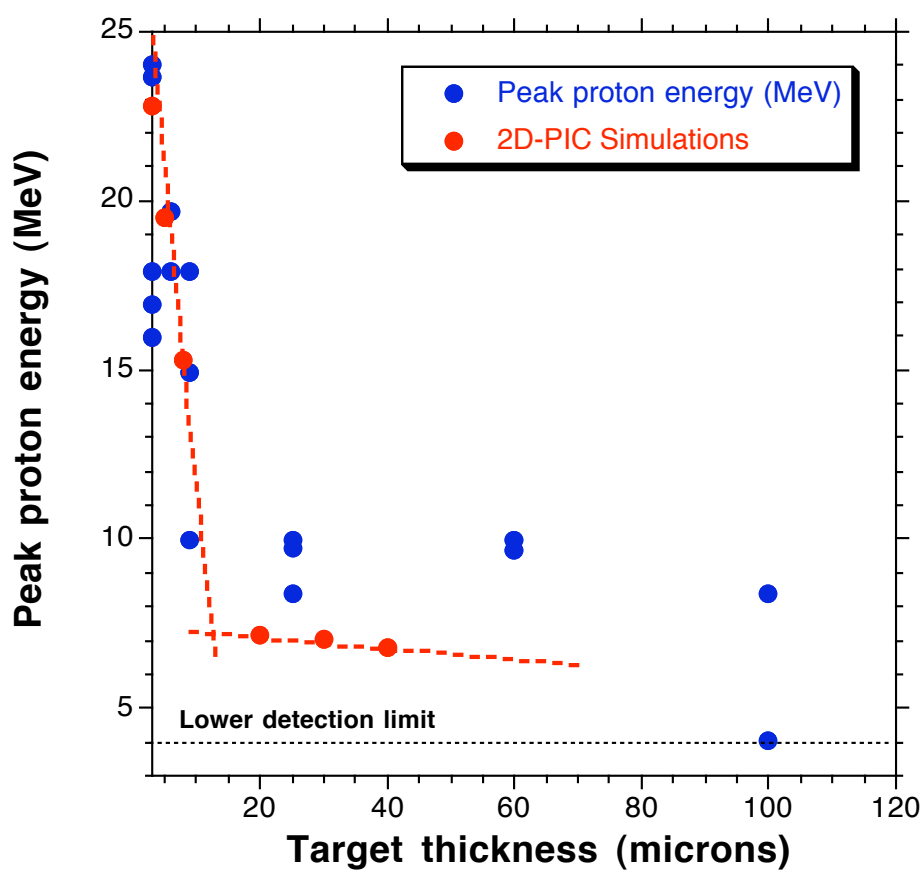

Figure 1. peak proton energy vs. target thickness. The abrupt change in slope is due to the enhancement of proton acceleration of refluxing electrons in the target. For target thickness less than 15microns refluxing effectively increases the field accelerating the protons. For targets large than 15 microns there is no net increase due to refluxing[5].

Physical Review Letters in 2002 [5]. Further collaborative work on JanUSP was carried out with scientists from Queens University, Belfast. This research successfully determined the location of the virtual source of protons and was published in Physical Review Letters in 2004 [6]. A later experiment, also published in Physical Review Letters then confirmed the physical source of the highest energy protons to be at the rear of the target [7.] Finally we discovered that curving the proton producing foil into a hemispherical shape allowed us to manipulate the proton beam to produce a focus far from the original foil surface. This work, published in Physical Review Letters in 2003, has many applications including using the protons to isochorically heat a solid density material [8].

The second part of the proposal investigated the limits of proton radiography in both cold and laser shocked targets. We found that Proton radiography using laser-accelerated protons has intrinsically very good spatial resolution. Edge features of a few microns could be resolved when point projection techniques were used to backlight wires, microballoons and metal foils. Monte-Carlo modeling of proton propagation through these targets showed that multiple scattering of the protons was responsible for image 
formation. The high resolution was due to the extremely good laminarity and very low emittance of the beam $[9,6]$.
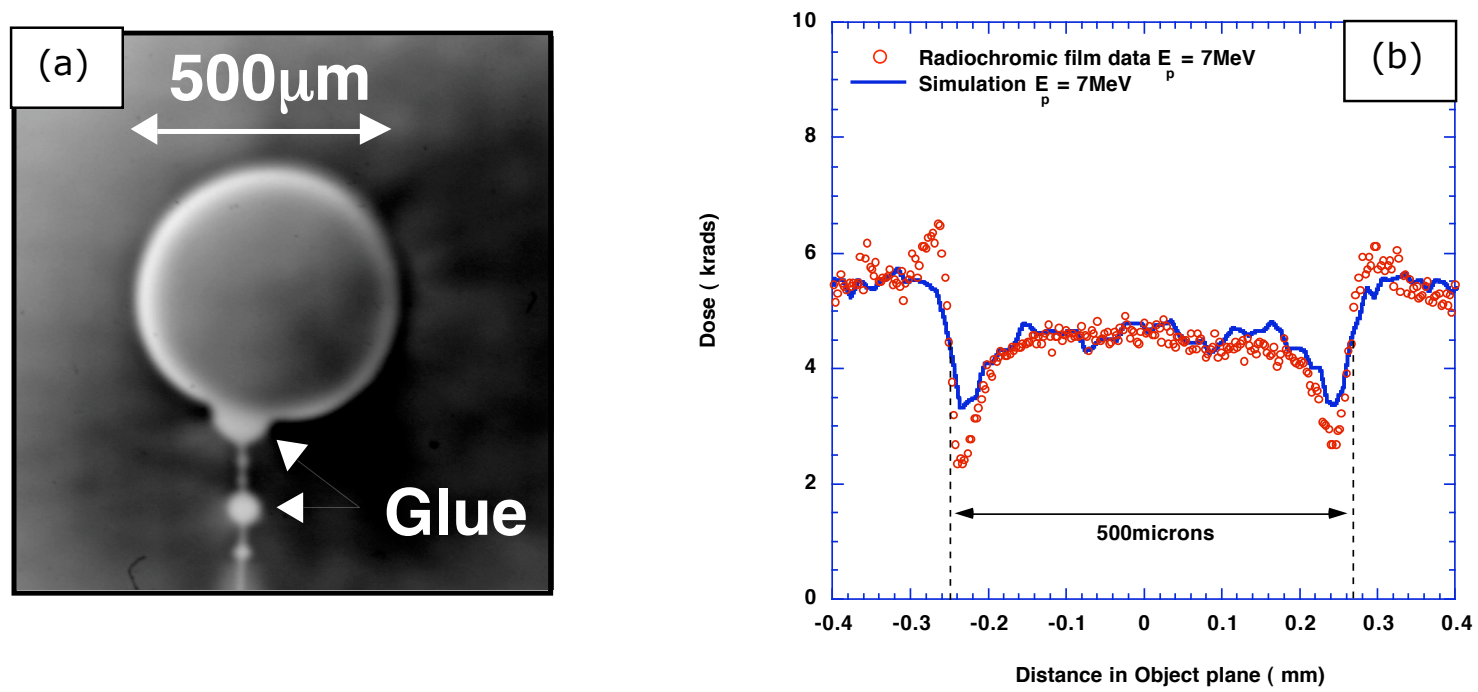

Fig.2(a) proton radiograph of a $500 \mathrm{~mm}$ microballoon,(b) lineout and Monte-Carlo simulation. The Simulation closely matches the data.

Point projection radiographs of ripple foils were also obtained during these experiments. The data showed that $4 \%$ density perturbations could be measured in $22 \mu \mathrm{m}$ thick aluminum foils. Thicker foils produce more multiple scattering for the same proton energy so this technique could be applicable for measuring density perturbations in thick high $\mathrm{Z}$ samples using higher proton energies. Simulations of these experiments is ongoing and the results will be written up in a publication for Review of scientific instruments. 
We applied these techniques to an laser driven implosion experiment, carried out on the Vulcan laser at the Rutherford Appleton Laboratory. Six beams of the laser were used implode a 500 $\mu \mathrm{m}$ diameter microballoon, while a synchronized short pulse beams was used to provide a proton radiograph at various times throughout the implosion. This work showed that high quality proton radiographs could be obtained throughout the whole time history of the implosion, including the stagnation point as shown in figure 3 . These results were used to benchmark Monte- Carlo simulations, which were then used to simulate the technique on a full scale NIF implosion. This work has been written up for submission to Physical Review Letters [10]. The same technique has also recently been applied to diagnosing density conditions in a target that has been shocked by a laser pulse. Proton radiographs were used to diagnose the density profile conditions following break-out and release of a shock in a planar target [11].

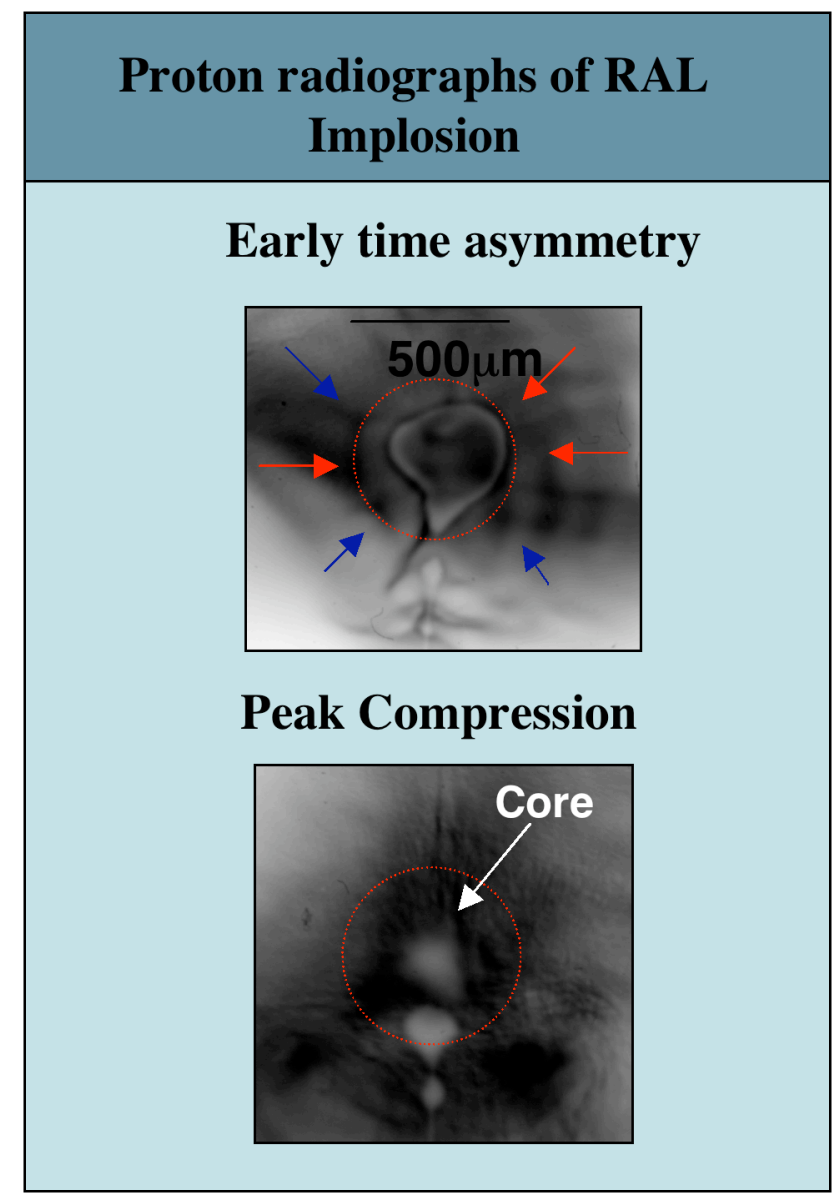

Figure 3 Top image shows a proton radiograph of an asymmetrically driven implosion. The resolution is good and the shell distortions are clearly visible. The bottom image shows a more symmetric implosion at a time close to stagnation. The capsule has assembled to a core withy density close to $3 \mathrm{~g} / \mathrm{cc}$. 
Finally we carried out a complex experiment on the LULI facility at Ecole Polytechnique, France. This experiment used proton deflectometry to measure electromagnetic fields in a laser-produced plasma. The technique originated from a suggestion to use Moiré techniques to quantitatively measure the proton deflection angles [12]. Experiments on JanUSP showed that Moiré techniques using double grids could be used with these laser produced protons [13]. The technique was successfully adapted by using a single grid in the LULI experiment.

\section{Proton probing of laser driven E fields}

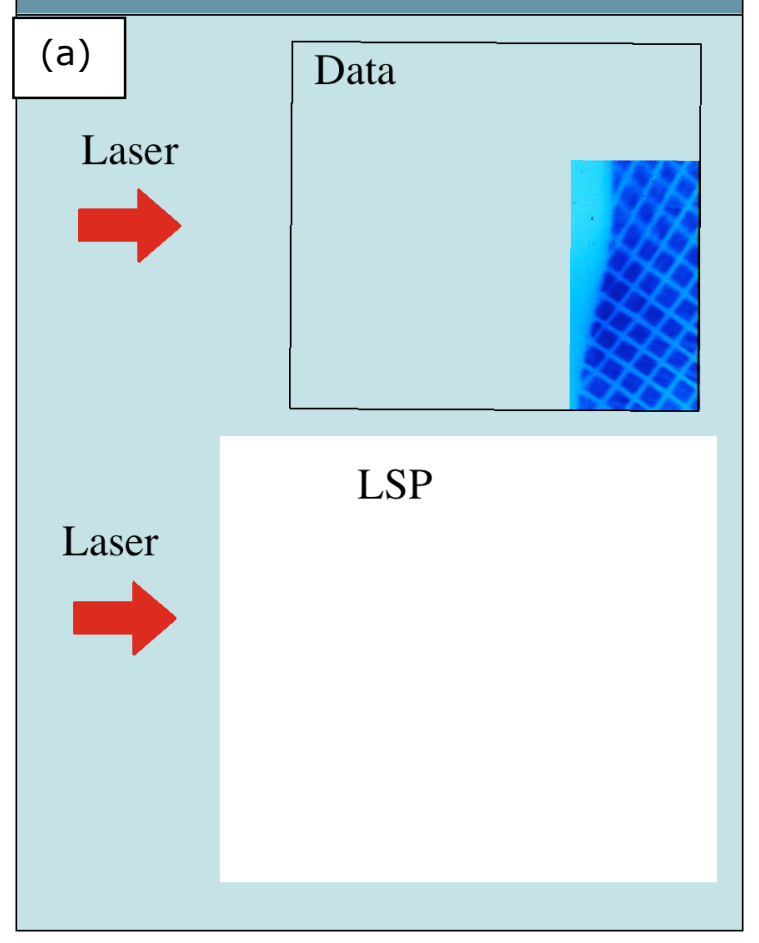

MG B fields in Ignition hohlraums can be readily measured using proton probing

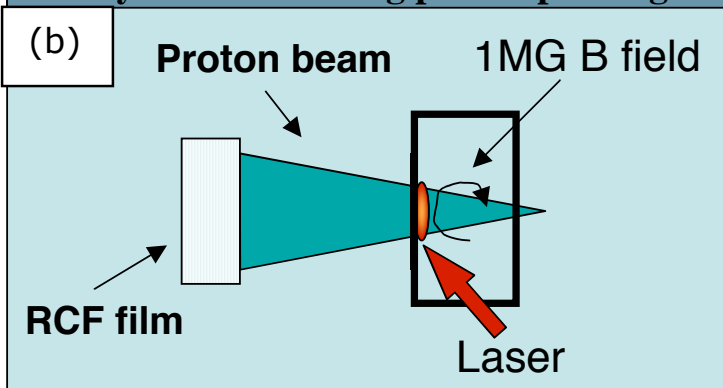

LSP simulated proton deflection

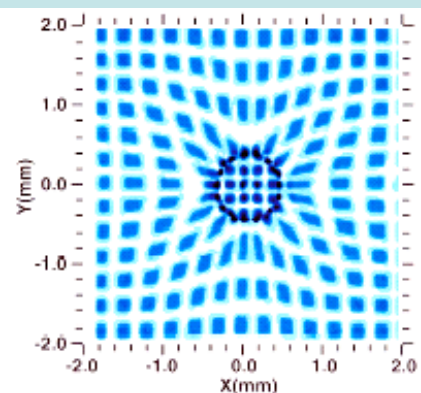

Figure 4 (a) Experimental deflectogram and LSP/LASNEX simulation of the data. The simulation shows the same overall shape and the pile up effect of protons at the edge of plasma but the magnitude of the deflection is lower than the experiment. (b) Simulated deflectogram of a conceptual experiment to measure magnetic fields in plasmas. The model is now being used in a predicative way to design new experiments.

The grids are used to impress a modulation on the proton beam before it goes through the plasma to be probed as shown in fig 4. A proton deflectogram from the experiment is shown at the top of fig 4(a). The effect of fields on the protons can then be quantitatively evaluated by measuring the distortions of the grid pattern as it traverses the field region. This work was used to compare to Lasnex simulations of the long pulse interaction. Lasnex was adapted to include source terms for electric and magnetic fields driven by pressure gradients in the plasma. A second particle code (LSP) was then used to 
determine the propagation of protons through the plasma field generated by lasnex. The plasma is the semi-spherical region in the middle of the image. It is clear from this image that the grid is very distorted inside the plasma, with around a factor two increase in the deflection angle of the proton beam. Another feature is the clear increase in proton signal around the edges of the hemispherical region due to a pile up of the protons here. The simulations successfully models both these effects, though the amount of distortion is less in the simulations than in the experimental data. These complex simulations are currently being used to determine if magnetic fields inside hohlraums can be characterized using proton probing, as is shown in fig. 4(b). In this particular case the $1 \mathrm{MG}$ torroidal magnetic field produces a focusing effect of the protons resulting in a very strong deflection. This work is currently being written up for submission to Science [14] and Physics of Plasmas [15].

In conclusion proton radiography is currently being considered as a diagnostic technique for NIF implosions and magnetic field measurements in NIF laser driven hohlraums. This project has led to many high quality publications and fostered important and lasting collaborations with numerous outside institutions. It has shown that proton radiography can successfully be implemented to measure densities and electromagnetic fields in plasmas that are particularly relevant for Inertial Confinement Fusion as well as other more general plasma conditions.

\section{References:}

1. N.S.P. King et al., Nuc. Instr. Methods Phys. Res A 42484

2. R. Snavely et al., Physical Review Letters, 852945 (2000). E. Clarke et al., Physical Review Letters, 84670 (2000).

3. M. Borghesi et al., Plasma Physics and Controlled fusion 43 A267, (2001).

4. J. Cobble et al., APL (2002).

5. A.J.Mackinnon et al., Physical Review Letters, 88215006 (2002).

6. M. Borghesi et al., Physical Review Letters, 92055003 (2004).

7. M. Allen et al., Physical Review Letters, 93265004 (2004).

8. P.K.Patel et al., Physical Review Letters, 91125004 (2003).

9. T.C.Cowan et al., Physical Review Letters, 92204801 (2004).

10. A.J.Mackinnon et al., preparation for submission to PRL.

11. M. Koenig et al., Nuc Fusion 44, S208 (2004).

12. P. Celliers Private communication (2001).

13. A.J.Mackinnon et al., APL (2003), ReV Sci Instruments (2004).

14. M. Borghesi et al., to be submitted to science

15. R. Town et al., to be submitted to Phys. of plasmas 